\title{
Fluidical bending actuator designed for soft octopus robot tentacle
}

\author{
Jan Fras ${ }^{1}$, Mateusz Macias ${ }^{2}$, Yohan $\mathrm{Noh}^{3}$ and Kaspar Althoefer ${ }^{1}$
}

\begin{abstract}
Soft actuation, due to its mechanical properties offer a complex motion that is not achievable for traditional mechanism. Thanks to that property it is often considered for bio-mimicking devices as many leaving creatures move by complex and distributed deformation. In this paper we propose a novel soft fluidical actuator designed to be used as a biomimicking tentacle in swimming octopus robot. The actuator has two degrees of freedom that enables bending in some range of directions and inherits the actuation effectiveness of single degree of freedom actuators while still able to control two of them. The actuator has been tested in terms of generated forces and motion capabilities and shows significant improvement regarding the state of the art actuators capable of completing the assumed task. The actuator enables the octopus robot to advance forward, change swimming directions and rotate around its primary axis. The paper presents the design, fabrication process and experimental verification of the proposed solution.
\end{abstract}

\section{INTRODUCTION}

\section{A. Motivation}

The actuator described in this paper has been designed in order to give an octopus robot the arms that are able to bend in some range of directions and to exert a significant force on the fluid that surrounds the robot in order to generate a sufficient amount of thrust. The expected bending motion has to be controlled in terms of curvature and bending plane direction. The octopus robot is presented in fig. 1 and has been described in more detail in [1].

\section{B. Survey on bending actuators}

The bending deformation is one of the commonly used motion types in soft robotics. Many designs are based on the this kind of motion deploying various types of actuators. Those actuators can be classified due to the working principle

\footnotetext{
*The research has received funding from the European Commission's project Horizon 2020 Research and Innovation Programme, project FourByThree under grant agreement No 637095.

${ }^{1}$ J. Fras and K. Althoefer are with the Centre for Advanced Robotics @ Queen Mary (ARQ), Faculty of Science and Engineering, Queen Mary University of London, London E1 4NS, UK j.fras @qmul.ac.uk, k.althoefer@qmul.ac.uk

${ }^{2} \mathrm{M}$. Macias is with Industrial Research Institute for Automation and Measurements, Warsaw, Poland macias@piap.pl

${ }^{3} \mathrm{Y}$. Noh is with the Department of Informatics, King's College London, London WC2R 2 LS, UK yohan. noh@kcl.ac.uk
}

and medium such as actuators powered by pressure [2], [3], tendon driven [4], temperature driven [5] or mixed [6]. In this work we consider the pressure based fluidical actuators, but even within this group some different actuation principles can be distinguished. First working principle is the bending due to unsymmetrical actuation [7]-[9]. In such case the structure of the actuator makes the acting forces to accumulate on some area of the device. This area elongates or shrinks more than the other side and consequently the device bends. It is worth noting that the pressure acting in the chambers can also reach negative values. An example of actuator working in negative range are vacuum actuators [9], [10]. The bending motion can be also caused by non-uniform rigidity [11]-[13] In such case one side of the actuator is less extensible than another so it elongates less when pressurized. Such devices can provide the pure bending if the less extensible side is not extensible at all [11] or combine the elongation and bending for the materials that still are able to extend [14]. Another principle is bending due to the geometry of the device. In [12] an actuator is described that have an symmetrical crosssection that is affected by the actuation pressure equally in all the points and uses no special layers of less extensible material, but is initially curved. Its shape is designed to bend when pressurized.

Each actuation strategy has some advantages, i.e. the first principle allows to house multiple actuation channels in the same part of the robot and gives the robot more than one degree of freedom (DOF). Consequently not only the curvature, but also the the bending direction, twist and elongation can be controlled. The second method in turn allows the robot to exploit the whole actuator cross-section to generate the motion and thus makes it more effective. However, the bending pattern can not be controlled and is defined during the manufacturing process [13].

The approach we propose in this paper combines those two methods in order to maximize the bending efficiency in the primary bending direction but introduce second degree of freedom for controlling the bending plane direction. 


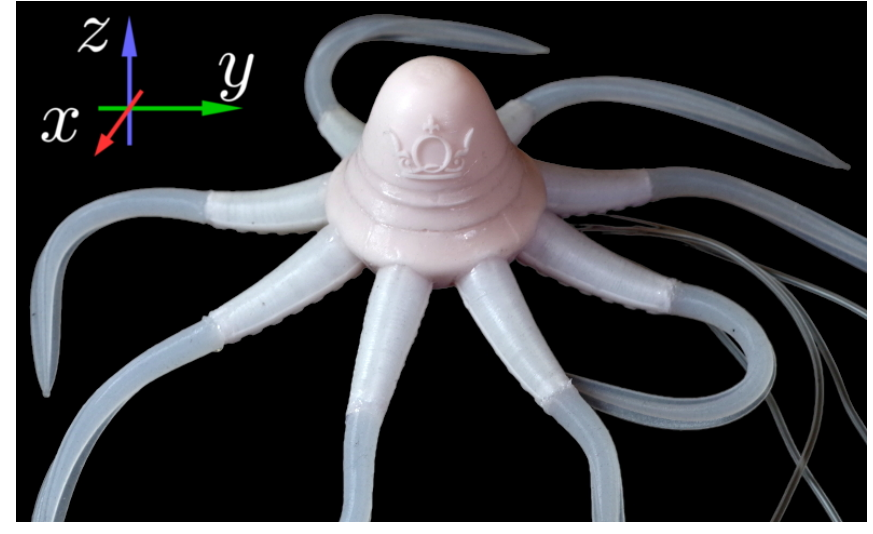

Fig. 1: The octopus robot powered by soft fluidical actuators. It consists 8 identical arms working in 2 groups. Each arm utilizes the actuator capable of bending in some range of directions and a passive part that is designed to generate the thrust when pushed by the active part of the tentacle.

\section{DESIGN}

\section{A. The robot overview}

The soft octopus robot is designed to swim in water and to be able to advance, turn and twist. For that reason the tentacles are internally connected and working in two independent groups. Each tentacle have two inner channels that enable it to bend in some range of directions. Symmetrical actuation of all the chambers of all the tentacles generates a coaxial thrust that pushes the robot forward. Actuation of one group only generates unsymmetrical thrust that pushes one side of the robot and consequently the robot turns. Providing pressure to one chamber per actuator causes them to bend to the side and this way affects the robot motion as well. Depending on the chosen pattern, one chamber actuation can result in rotational or twisting motion. Different actuation patterns have been presented in fig. 2

\section{B. The actuator design}

The arm of the robot consists of two parts. The active part housing the actuator that generates the bending motion, and a passive part that follows the motion enforced by the active part. The active part is assumed to generate the arm motion only, and the passive part to generate the thrust. The design of the arm is presented in fig. 3 .

The construction of the actuator described in this paper is derived from actuation chambers used in STIFF-FLOP manipulator [7]. The bending mechanism is, however, inspired by the previous work made on soft compliant graspers and hands presented in [14], [15]. In order to enable the

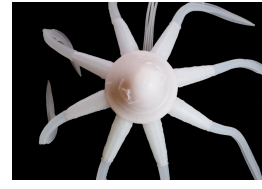

(a)

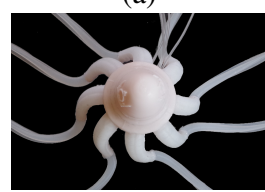

(d)

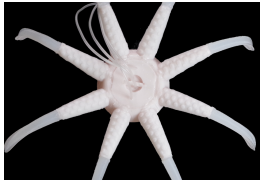

(b)

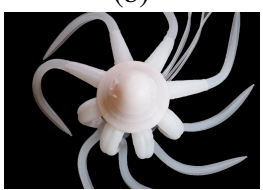

(e)

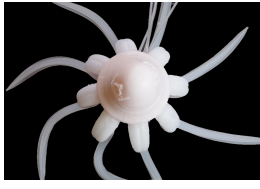

(c)

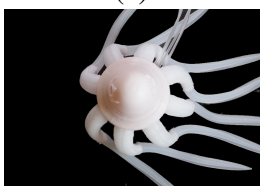

(f)
Fig. 2: Various actuation patterns. (a),(b) - passive robot, top and bottom view, respectively, (c) - same pressure for all actuation chambers, forward movement, (d) - axial activation symmetry, rotation along $z$ axis (twist), (e) - one side activation, rotation around $y$ axis (turn), (f) - two sides activation, rotation around $x$ axis (turn). Frame of reference is presented in fig. 1 .

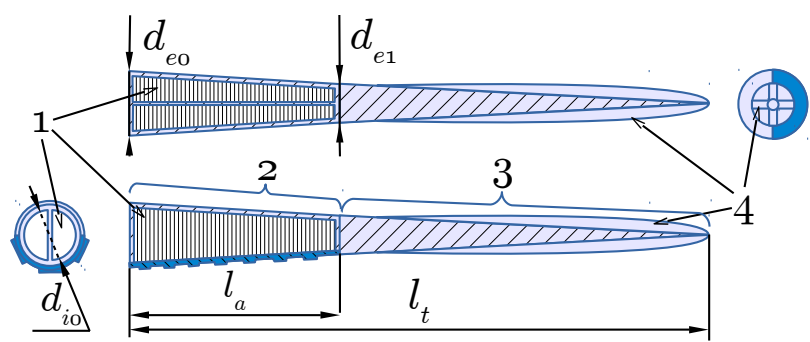

Fig. 3: The robot's arm design. 1 - actuation chambers, 2 and 3 - active and passive parts of the arm, consequently, 4 - fin-like surfaces. $l_{a}=40 \mathrm{~mm}, l_{t}=140 \mathrm{~mm}, d_{i 0}=10 \mathrm{~mm}$, $d_{e 0}=15 \mathrm{~mm}, d_{e 1}=9 \mathrm{~mm}$.

assumed motion the actuator consists of two independent pressure chambers that tend to elongate when pressurized. The inner side of the actuator is covered with stiffer silicone. Due to mechanical properties, that layer elongates less than the rest of the actuator when pressurized resulting in bending. The actuation chambers are constrained by a reinforcement formed with polyester thread as a tight helix integrated into their walls. Such an reinforcement strategy does not interfere the elongation capabilities of the actuator while efficiently constrains any radial deformation such as ballooning. The actuator structure is presented in fig. 4. The used materials are SmoothOn EcoFlex 00-30 and SmoothOn Smooth Sil 
940 silicones for as softer and stiffer material respectively.

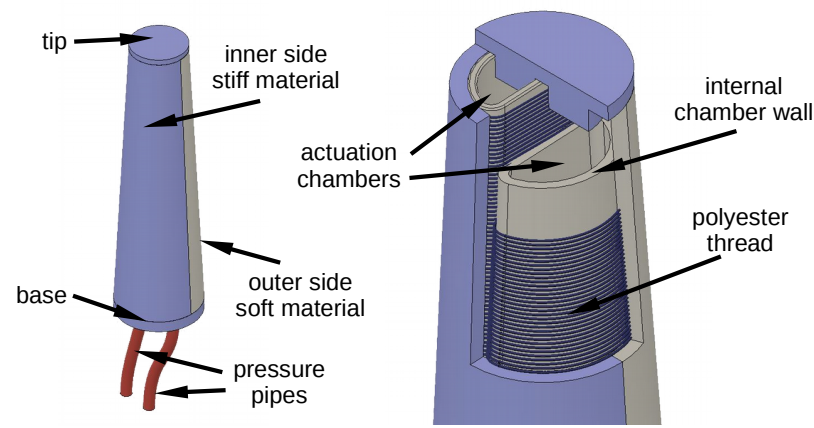

Fig. 4: The actuator structure. Actuation chambers with reinforcement visible. Stiff silicone denoted in blue, soft in gray colour.

The inner layer of stiffer material enforces the bending motion of the device when a pressure is applied, however independent actuation of each pressure chamber influences the direction of bending plane. The chamber that is pressurized with higher pressure value tends to elongate more that the another one, thus pushes the corresponding side of the device towards the other side. The novel aspect of this actuator is that other bending actuators that provide bending plane control are usually deploying several actuation structures and the bending is resulting from unsymmetrical actuation. Since, in such case the bending motion requires some actuators to be actuated less than others, the overall actuation area is fairly limited [7], [8]. The proposed actuator utilizes the whole actuation area to provide a bending, which results in high efficiency, while the bending plane still can be controlled by varying the pressure values in both sides of the actuator. The actuator response for different pressure inputs is presented in fig. 5 .

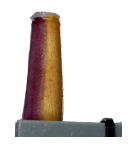

(a)

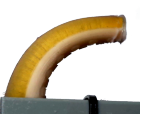

(b)

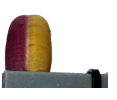

(c)

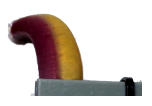

(d)

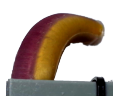

(e)
Fig. 5: The actuator, a) passive, b) and c) symmetrically bended, pressures equal, side view and top view respectively, d) bended to the left, right chamber pressure higher than left, e) bended to the right, left chamber pressure higher than right.

\section{MANUFACTURING}

The actuator is made of silicone material cast in 3Dprinted moulds produced with desktop 3D printer, Zortrax $\mathrm{m} 200$. The process consists of several stages as described below.

1) Step I, reinforcement: As the initial step the reinforcement is formed. It is made of a polyester thread using a dedicated 3D printed conical core. Each actuator requires two separate reinforcement structures. The reinforcement is formed manually using cordless that helps to control pitch and tension of the thread, ??.

2) Step IIa, external layer, soft side: The mould cores with the thread deployed are then inserted into an external mould and soft silicone is injected. Due to the mould design, only one half of the actuator gets covered with the silicone material while the another remains untouched. After the silicone cures the mould is open and the actuator is moved to another mould to create the missing part of the external layer.

3) Step IIb, external layer, stiff side: When the mould is exchanged, stiffer silicone is injected in order to create the another half of the external layer. When it is cured, the mould is open and the cores are gently removed. Since the cores are conical they can be easily removed towards the wider end of the actuator. At that moment the external layer of the actuator is ready with the reinforcement structure attached to its internal side, fig. $6 \mathrm{~b}$.

4) Step III, internal layer: The reinforcement structure is already attached to the actuator, however, it is still very fragile since it is not fully integrated with the silicone. Applying any pressure would make the silicone structure to extend radially and detach the thread from the silicone surface. For that reason the actuator requires another moulding step to fully integrate the thread from the silicone. For that reason a smaller semi-conical silicone structure is created using another set of moulds. That structure is then attached inside the reinforcement using the same kind of silicone fig. $6 \mathrm{~d}$.

5) Step IV, closure: When the internal layer is attached, the body of the actuator is finished. For the last step the tip and the base of the actuator are sealed with stiffer material, the same used for the internal side of the actuator. In the bottom layer small wholes are created in order to attach small pressure pipes providing the actuation fluid.

\section{EXPERIMENTAL VERIFICATION AND RESULTS}

The robustness of the proposed solution has been verified experimentally. For that purpose various versions of the actuator have been manufactured that includes the proposed actuator with two semi-conical chambers and stiffer internal 


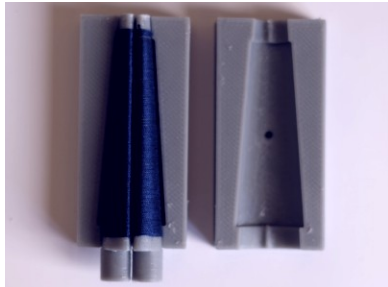

(a)

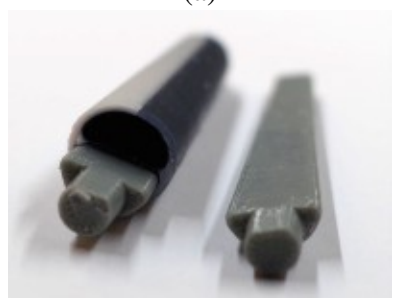

(c)

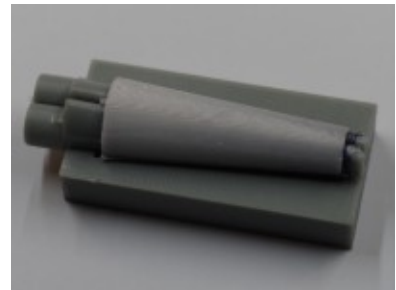

(b)

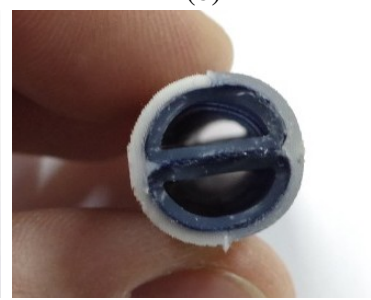

(d)
Fig. 6: Fabrication steps: (a) - reinforcement, (b) - external layer, (c) - reinforcement attached to external layer exposed, , (d) - internal chamber layers attached.

layer (fig. 7a), an actuator with one actuation chamber only (fig. 7b) and actuator with two cylindrical chambers shifted towards the outer actuator's side (fig. 7c). The actuator with single actuation chamber is expected to generate more bending and exert higher forces than the proposed one, since its actuation volume is bigger due to the volume taken for chambers separation in the proposed actuator. The drawback is that the bending direction in that case can not be controlled. The actuator with shifted chambers bends due to unsymmetrical actuation and for that reason the stiffer outer layer has been substituted with a layer of the same silicone as the rest of the actuator. This actuator enables the bending direction control based on the same principle as the proposed one, but due to significantly reduced actuation area is expected to exert less force and bending compared to other actuators. The actuators and their cross-sections are presented in fig. 7.

For the experiment a simple vision system has been used fig. 8a [12]. The actuation process of all the actuators has been recorded and using image processing the pressure value and the bending angle had been exerted form the video. The actuation in range from 0 to 0.4 bar had been repeated for each actuator for 6 times. The average actuation curves with statistical errors are presented in fig. 9. For the force characterization the force value had been measured at the actuator tip using a $3 \mathrm{~d}$ printed optical force sensor [16], fig. 8b. The resulting plots are presented in fig. 10 .

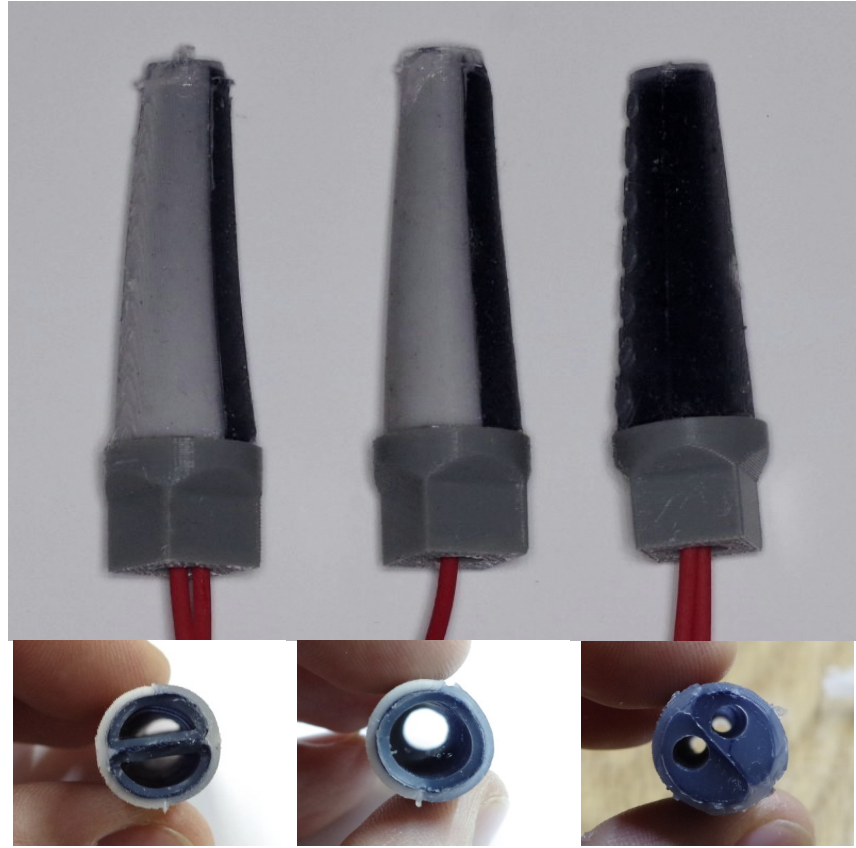

(a)

(b)

(c)

Fig. 7: Tested actuators (a) - proposed actuator, (b) - single DOF actuator, (c) - actuator based on unsymmetrical actuation. All the actuators have same dimensions.

The measured deviation of bending plane direction for the proposed actuator is approximately \pm 28 degrees for pressure equal 0.4 bar applied to one chamber only.

\section{DisCUSSION AND CONCLUSIONS}

The experimental data shows that the most effective bending is provided by single DOF actuator, fig. $7 \mathrm{~b}$. It is due to the biggest possible actuation volume. The proposed actuator bends less for the same amount of pressure, as part of the actuation area is occupied by the wall in between the actuation chambers, fig. 7a. Such a wall introduces also additional material that is stretched during actuation process and limits the bending deformation even further. The lowest bending efficiency is provided by the unsymmetrical actuator geometry, fig. $7 \mathrm{c}$, as in that case the actuation volume is the lowest while the amount of stretched material the biggest. For the same reason the unsymmetrical actuator generates less force than the other ones. The experimental verification shows that the proposed actuator provides an comparable efficiency with single DOF actuators in therms of generated force. The bending characteristics is slightly less effective than the one of single DOF actuator. In the same time it 


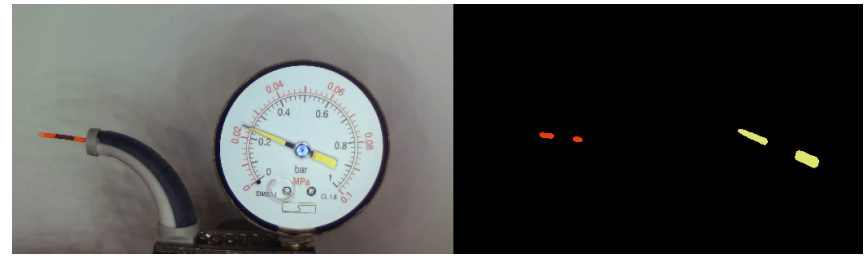

(a)

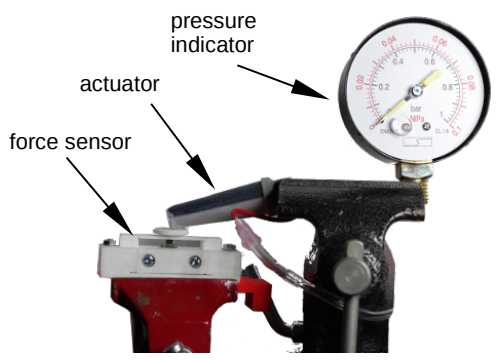

(b)

Fig. 8: Bending and force measurement, (a) captured and processed frame, (b) force measurement setup.

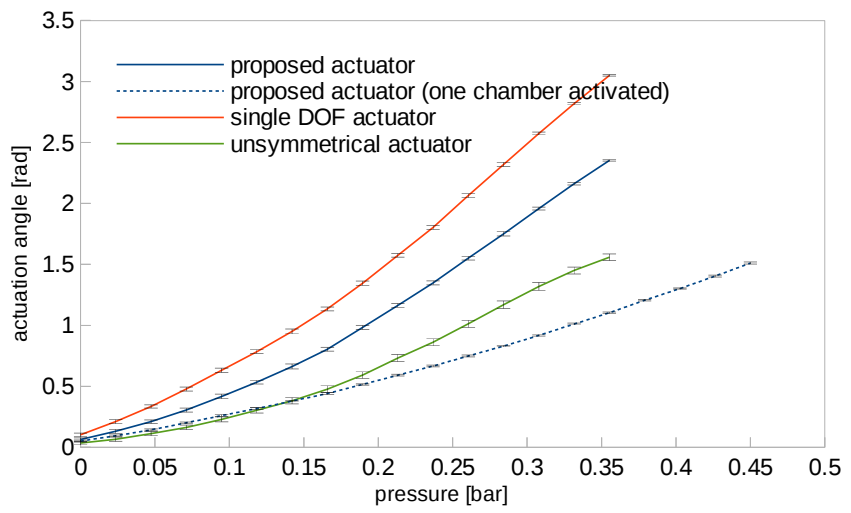

Fig. 9: Bending vs pressure characteristics of tested actuators.

provides the second degree of freedom while overperforming unsymmetrical actuator that is capable of the similar kind of motion.

\section{REFERENCES}

[1] J. Fras, Y. Noh, Macias M., H Wurdemann, and K. Althoefer. Bioinspired octopus robot based on novel soft fluidic actuator. In submitted to International Conference on Robotics and Automation. IEEE, 2018.

[2] Panagiotis Polygerinos, Zheng Wang, Kevin C Galloway, Robert J Wood, and Conor J Walsh. Soft robotic glove for combined assistance and at-home rehabilitation. Robotics and Autonomous Systems, 73:135-143, 2015.

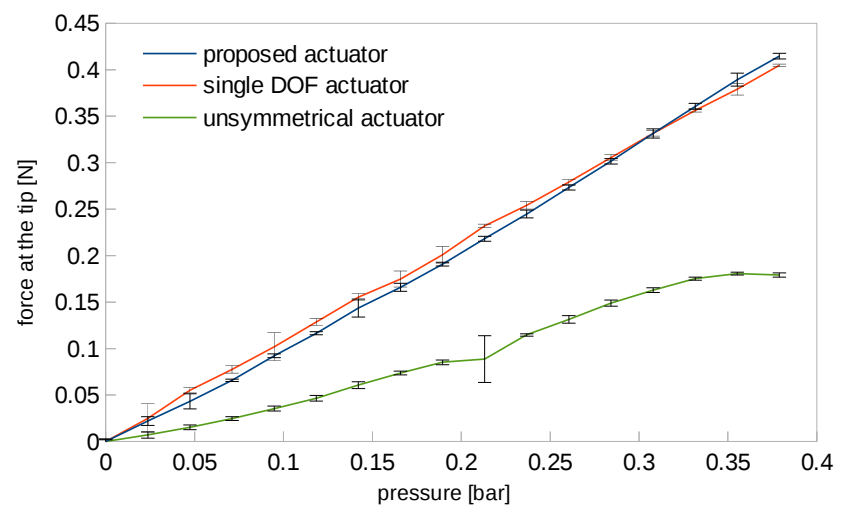

Fig. 10: Force vs pressure characteristics for the proposed actuator, single DOF actuator and actuator based on unsymmetrical actuation.

[3] Y. Sun, Y. S. Song, and J. Paik. Characterization of silicone rubber based soft pneumatic actuators. In International Conference on Intelligent Robots and Systems, pages 4446-4453, Nov 2013.

[4] Ian D Walker. Continuous backbone "continuum" robot manipulators. ISRN Robotics, 2013, 2013.

[5] Aslan Miriyev, Kenneth Stack, and Hod Lipson. Soft material for soft actuators. Nature Communications, 8(1):596, 2017.

[6] A. Stilli, H. A Wurdemann, and K. Althoefer. Shrinkable, stiffnesscontrollable soft manipulator based on a bio-inspired antagonistic actuation principle. In International Conference on Intelligent Robots and Systems, pages 2476-2481. IEEE, 2014.

[7] J. Fras, J. Czarnowski, M. Macias, J. Glowka, M. Cianchetti, and A. Menciassi. New stiff-flop module construction idea for improved actuation and sensing. In International Conference on Robotics and Automation, pages 2901-2906. IEEE, 2015.

[8] K. Suzumori, S. Iikura, and H. Tanaka. Development of flexible microactuator and its applications to robotic mechanisms. In International Conference on Robotics and Automation, pages 1622-1627. IEEE, 1991.

[9] Matthew A Robertson and Jamie Paik. New soft robots really suck: Vacuum-powered systems empower diverse capabilities. Science Robotics, 2(9):eaan6357, 2017.

[10] Shuguang Li, Daniel M. Vogt, Daniela Rus, and Robert J. Wood. Fluiddriven origami-inspired artificial muscles. Proceedings of the National Academy of Sciences, 2017.

[11] Raphael Deimel and Oliver Brock. A novel type of compliant and underactuated robotic hand for dexterous grasping. The International Journal of Robotics Research, 35(1-3):161-185, 2016.

[12] J. Fras, Y. Noh, H Wurdemann, and K. Althoefer. Soft fluidic rotary actuator with improved actuation properties. In International Conference on Intelligent Robots and Systems. IEEE, 2017.

[13] Kevin C Galloway, Panagiotis Polygerinos, Conor J Walsh, and Robert J Wood. Mechanically programmable bend radius for fiberreinforced soft actuators. In Advanced Robotics (ICAR), 2013 16th International Conference on, pages 1-6. IEEE, 2013.

[14] J. Fraś, M. Maciaś, F. Czubaczyński, P. Salek, and J. Glowka. Soft flexible gripper design, characterization and application. In International Conference SCIT, Warsaw, Poland. Springer, 2016.

[15] J. Fraś and K. Althoefer. Bio-mimetic pneumatic soft prosthetic hand. In UK-RAS Conference on 'Robotics and Autonomous Systems', Bristol. UK-RAS, 2017. 
[16] Y. Noh, Akihiro Shimomura, Masanao Segawa, Hiroyuki Ishii, J. Solis, Atsuo Takanishi, and Kazuyuki Hatake. Development of tension/compression detection sensor system designed to acquire quantitative force information while training the airway management task. In 2009 IEEE/ASME International Conference on Advanced Intelligent Mechatronics, pages 1264-1269, July 2009. 